\title{
Spreading improvements for advanced COPD care through a Canadian Collaborative
}

\author{
This article was published in the following Dove Press journal: \\ International Journal of COPD \\ 26 July 2017 \\ Number of times this article has been viewed
}

\section{Graeme M Rocker' \\ Claudia Amar ${ }^{2}$ \\ Wendy L Laframboise ${ }^{3}$ \\ Jane Burns ${ }^{4}$ \\ Jennifer Y Verma ${ }^{2}$}

'Division of Respirology, Nova Scotia Health Authority/Dalhousie University, Halifax, NS, ${ }^{2}$ Canadian Foundation for Healthcare Improvement, ${ }^{3}$ The Ottawa Hospital COPD Outreach Program, Ottawa, ON, ${ }^{4}$ Providence COPD Outreach Program, Vancouver, BC, Canada
Correspondence: Graeme M Rocker Division of Respirology Nova Scotia Health Authority/Dalhousie University, 4457 Halifax Infirmary, 1796 Summer St, Halifax, NS B3H 3A7, Canada

Tel + I 90247366 II

Fax +I 9024736202

Email gmrocker@dal.ca
Background: A year-long pan-Canadian quality improvement collaborative (QIC) led by the Canadian Foundation for Healthcare Improvement (CFHI) supported the spread of the successful Halifax, Nova Scotia-based INSPIRED COPD Outreach Program ${ }^{\mathrm{TM}}$ to 19 teams in the 10 Canadian provinces. We describe QIC results, addressing two main questions: 1) Can the results of the Nova Scotia INSPIRED model be replicated elsewhere in Canada? 2) How did the teams implement and evaluate their versions of the INSPIRED program?

Methods: Collaborative faculty selected measures that were evidence-based, relatively simple to collect, and relevant to local context. Chosen process and outcome measures are related to four quality domains: 1) patient- and family-centeredness, 2) coordination, 3) efficiency, and 4) appropriateness. Evaluation of a complex intervention followed a mixed-methods approach.

Results: Most participants were nurse managers and/or COPD educators. Only $8 \%$ were physicians. Fifteen teams incorporated all core INSPIRED interventions. All teams carried out evaluation. Thirteen teams actively involved patients and families in customized, direct care planning, eg, asking them to complete evaluative surveys and/or conducting interviews. Patients consistently reported greater self-confidence in symptom management, a return to daily activities, and improvements to quality of life. Twelve teams collected data on care transitions using the validated three-item Care Transitions Measure (CTM-3). Twelve teams used the Lung Information Needs Questionnaire (LINQ). Admissions, emergency room visits, and patient-related costs fell substantially for two teams described in detail (combined enrollment 208 patients). Most teams reported gaining deeper knowledge around complexities of COPD care, optimizing patient care through action plans, self-management support, psychosocial support, advance care planning, and coordinating community partnerships.

Conclusion: Quality-of-care gains are achievable in the short term among different teams across diverse geographical and social contexts. A well-designed, adequately funded publicprivate partnership can deliver widespread beneficial outcomes for the health care system and for those living with advanced COPD.

Keywords: INSPIRED COPD Outreach Program ${ }^{\mathrm{TM}}$, quality improvement, quality improvement collaborative, admission/readmission

\section{Introduction}

Advanced COPD is characterized by frequent acute exacerbations (AECOPDs), a leading cause for emergency department (ED) visits and hospital admissions across Canada. ${ }^{1,2}$ Admissions due to AECOPD account for CAD $\$ 750$ million/year in hospitalbased costs. ${ }^{3}$ Beyond debilitating dyspnea in advanced stages, many patients struggle with emotional, psychological, and existential concerns related to loss of meaning, hope, and fear of death. ${ }^{4}$ 
To address gaps between care needed and provided, ${ }^{5}$ the INSPIRED COPD Outreach Program ${ }^{\mathrm{TM}}$ was implemented in Nova Scotia in 2010 as an evidence- and communitybased holistic approach to COPD care beyond a hospital admission. ${ }^{6,7}$ INSPIRED enables patient- and family-centered care and support across care transitions for those living with advanced COPD. Patients are followed post-discharge at home, through two visits by a certified respiratory educator (CRE) providing self-management support ${ }^{8,9}$ individualized action plans for AECOPDs and referrals to allied health professionals. To respond to previously documented distress, ${ }^{10}$ a spiritual care practitioner (SCP) provides psychosocial support and advance care planning (ACP) through two additional visits. Patients have access to a telephone line during working hours and are followed beyond completion of visits by telephone contact at monthly intervals over 3 months and then a final call at 12 months.

INSPIRED has positively impacted clinical quality and $\operatorname{costs}^{11}$ while improving patient and family experience. ${ }^{6,7}$ Only 26 patients need to complete the program to avert CAD $\$ 100,000$ of health care spending, after deduction of program costs. ${ }^{11}$ If INSPIRED reached $<1 \%$ of people living with COPD in Canada, an independent economic analysis estimated 68,500 fewer ED visits, 44,100 fewer hospitalizations, and 400,000 fewer bed days over the next 5 years. Net of program expenses approximates to aversion of $\sim$ CAD $\$ 688$ million in hospital-based costs. ${ }^{12}$

The Advisory Panel on Healthcare Innovation recently attested to the need for a stable funder to turn a successful regional pilot into a successful national initiative. ${ }^{13}$ We describe how the year-long pan-Canadian quality improvement collaborative (QIC) led by the Canadian Foundation for Healthcare Improvement (CFHI), a federally funded non-profit organization dedicated to spreading health care improvement, supported the spread of the INSPIRED model to 19 teams in the 10 Canadian provinces. ${ }^{14,15}$ We describe some quality improvement results, addressing two main questions:

1. Can the results of the Nova Scotia INSPIRED model be replicated by other health care delivery organizations across Canada?

2. How did the teams organize themselves to implement and evaluate their own versions of the INSPIRED program?

\section{Methods}

At the outset of the collaborative, teams identified the population for which the anticipated interventions would be most impactful. Table 1 presents a list of the most common
Table I Most common patient inclusion/exclusion criteria across the 19 INSPIRED teams

\begin{tabular}{ll}
\hline Inclusion criteria & Exclusion criteria \\
\hline With moderate-severe COPD diagnosis & Living in long-term \\
(3-5 on the MRC dyspnea scale) & care facility \\
One or more ED visit(s) and/or admission(s) & Cognitive impairment \\
to hospital in the past year & \\
Living in a defined catchment area & Life expectancy of \\
Community dwelling & 6 months or less \\
Able and willing to participate & \\
\hline
\end{tabular}

Abbreviations: MRC, Medical Research Council; ED, emergency department.

patient inclusion and exclusion criteria teams used to guide enrollment. We have described the Halifax INSPIRED enrollment criteria in detail elsewhere. ${ }^{16,17}$

Teams were encouraged to prioritize patients with moderate to severe COPD. This was not feasible for all teams given the prevalence of co-morbidity among patients living with advanced COPD. In many cases, the interventions encompassed care for complex patients dealing with a multitude of acute and chronic health and social issues.

Faculty associated with the collaborative worked with CFHI to select measures that were evidence-based, relatively simple to collect, and relevant to local planned interventions. Teams collected process and outcome measures related to four quality domains: 1) patient- and family-centeredness, 2) coordination, 3) efficiency, and 4) appropriateness.

Regarding patient and family-centeredness, some teams collected qualitative data using the Hopes and Expectations Questionnaire, a five-item questionnaire used in a recent Halifax study ${ }^{16}$ that assesses patients' expectations pre-post INSPIRED. To measure changes in the quality of coordination of care, teams used the validated three-item Care Transitions Measure (CTM-3).$^{18}$ For efficiency, teams variably tracked ED visit rate, hospitalization rate, unplanned readmissions post-discharge, and lengths of stay. To measure appropriateness of care, teams identified and tracked a minimum of two metrics aligned with their specific interventions (Table 2). As a quality improvement initiative, formal

Table 2 Measures recorded to assess appropriateness of care

\begin{tabular}{ll}
\hline Measures & Teams evaluating (n) \\
\hline Patients receiving smoking cessation advice & 2 \\
Assessed/monitored by CRE upon admission & 2 \\
Action plan developed at discharge & 9 \\
Referred to pulmonary rehabilitation & 3 \\
Appropriate follow-up care & 6 \\
Spirometry to confirm diagnosis & 2 \\
\hline
\end{tabular}

Abbreviation: CRE, certified respiratory educator. 
research ethics board (REB) approval was neither required nor sought by CFHI.

\section{Analysis}

Evaluation of process related to a complex intervention should encompass both quantitative and qualitative assessments as recently confirmed in a 2015 report from the Medical Research Council. ${ }^{19}$ Accordingly, we used a mixed-methods approach as we have in other related research studies. ${ }^{16,20}$

\section{Results}

We present results under the following domains: team composition, team adaption of the INSPIRED model of care, how teams adapted the evaluation framework to better meet local needs, results achieved against the four quality domains, and the main barriers that impacted teams' overall results.

\section{Improvement team composition}

Over the course of the collaborative, 19 inter-professional teams representing 78 organizations and $>214$ health care professionals adapted the INSPIRED model of care. Most participants were clinical administrative leads, eg, nurse managers and COPD educators. Only $8 \%$ of participating members were physicians (reflecting a model of care that relied on an inter-professional team working to their full scope of practice to deliver the suite of interventions).

\section{Adaptations of the INSPIRED model of care}

Participating organizations adapted the INSPIRED model of care in a variety of ways. Overall, 15 (79\%) teams incorporated all core INSPIRED interventions (Table 3), while $13(68 \%)$ incorporated additional interventions such as smoking cessation, pulmonary rehabilitation, and improving inter-professional collaboration for patients living with complex needs.

The providers responsible for delivering specific interventions varied by team who were free to rely on different professionals to adapt INSPIRED to local context and resources. For example:

- For the 18/19 teams that carried out the self-management support role, it was undertaken by registered nurses (RNs)/CREs/registered respiratory therapists (RRTs) (14 teams), a physiotherapist (two teams), or a social worker (two teams).

- For the 16/19 teams providing a psychosocial support role, it was undertaken by a social worker (seven teams), RNs/CREs (five teams), or SCP (four teams). Of the 19 teams involved, 13 indicated they did not include or have access to an SCP.

- All teams carried out evaluation, but there were more evaluators $(n=22)$ than teams with the evaluation conducted by an RN/CRE/RRT (eight teams), a designated measurement lead (five teams), a utilization specialist or quality improvement lead (four teams), or another team member (five teams).

\section{Team adaptations of the measurement framework}

The collaborative was an opportunity to learn from and evolve the original INSPIRED model by using new metrics to track the effectiveness of interventions within a customized approach to local needs. For example, 12 teams collected data on care transitions using the validated CTM-3. ${ }^{18}$ Several teams $(n=12)$ used the Lung Information Needs Questionnaire (LINQ), ${ }^{21}$ a 20 -item survey designed to help clinicians

Table 3 INSPIRED collaborative intervention completion data across the 19 teams

\begin{tabular}{|c|c|c|c|c|}
\hline INSPIRED COPD Outreach Program ${ }^{\mathrm{TM}}$ interventions & $\begin{array}{l}\text { Teams } \\
\text { completed }(\mathbf{n})\end{array}$ & $\begin{array}{l}\text { Teams in } \\
\text { progress }(\mathbf{n})\end{array}$ & $\begin{array}{l}\text { Teams } \\
\text { planning (n) }\end{array}$ & $\begin{array}{l}\text { Not applicable/ } \\
\text { no data }\end{array}$ \\
\hline Optimization of medications and action plan prescription (prior to discharge) & II & 5 & - & 3 \\
\hline (In-home) self-management education by a certified COPD educator & 8 & 8 & - & 3 \\
\hline (In-home) psychosocial/spiritual support & 8 & 4 & I & 6 \\
\hline (In-home) advance care planning & 9 & 5 & I & 4 \\
\hline Phone access to team support (during working hours) & 10 & 6 & I & 2 \\
\hline $\begin{array}{l}\text { Monthly COPD educator phone follow-up for (at least) } 3 \text { months after } \\
\text { scheduled education visits }\end{array}$ & 8 & 7 & I & 3 \\
\hline $\begin{array}{l}\text { Liaison and partnership building with community and allied health care } \\
\text { support services }\end{array}$ & 6 & 12 & - & I \\
\hline Monitoring/evaluation for quality assurance purposes & 4 & II & - & 4 \\
\hline $\begin{array}{l}\text { Additional interventions (eg, smoking cessation, pulmonary rehabilitation, } \\
\text { and improving inter-professional collaboration for patients living with } \\
\text { complex need) }\end{array}$ & 7 & 3 & - & 9 \\
\hline
\end{tabular}

Note: '-' indicates not planning. 
identify which patients would be helped by information and the type of information an individual patient needs. Four teams used the COPD Assessment Tool (CAT), ${ }^{22}$ which assesses the impact of COPD on a patient's health status, and five teams chose a version of the Hopes and Expectation tool used in Halifax. ${ }^{16}$ Teams used a variety of outcome measures to assess efficiency of health care, eg, ED visit rate $(n=12)$, average length of stay ( $n=13)$, and unplanned AECOPD readmissions $(n=12)$. In the same way, teams relied on a variety of measures to assess appropriateness of care (Table 2).

\section{Patient and family outcomes and impact}

Thirteen teams actively sought to involve patients, families, and caregivers in the initiatives, most commonly involving them in customized, direct care planning, eg, asking them to complete evaluative surveys and/or conducting interviews to gain a deeper understanding of their care experiences. Family members and caregivers of enrolled patients commonly reported they felt better-supported throughout the hospital-tohome care transition. They also had the necessary information to help their loved ones better manage their COPD.

Patients who participated in an INSPIRED program within the collaborative have consistently reported 1) greater self-confidence in symptom management, 2) a return to daily activities, and 3) improvements to quality of life as highlighted by these quotes (Box 1).

\section{Provider and health system outcomes and impacts}

Teams achieved significant results related to patient- and family-centered care, coordination of care, appropriateness of care, and efficiency of care. In recognition that teams began the collaborative from quite differing levels of maturity of COPD care, we have chosen to describe some specific examples.

\section{Patient- and family-centered care}

Most teams reported a better understanding of the importance of the need for collaboration to seamlessly integrate the continuum of care and a more solid grasp of patients' actual needs. As an example, from the 12 teams who chose to use the LINQ tool, 18 of 20 patients enrolled at Joseph Brant Hospital (JBH) in Burlington, Ontario, showed improvement in their LINQ scores after participation in the program (mean difference 3.6 [95\% CI 1.9-5.3], $P=0.0003$ ).

\section{Coordination of care}

Teams developed systems and pathways for COPD patients to support their transition back to home and community. Among 41 patients enrolled at JBH completing pre-post scores, CTM-3 improved from mean (SD) 9.91 (1.36) to 11.21 (0.87), $P<0.0001$. Thirty-five patients improved (median improvement 20\%) indicating greater quality-ofcare transitions from hospital to home. Teams also became more aware of the potential benefits of working closely with community resources.

London Health Sciences Centre became more aware "of the extent of poverty within the city and how it impacts a person's ability to improve chronic disease management skills" as well as "truly getting to know the disease as well as empathizing with patients, families and caregivers."

In another example, Hamilton Health Sciences (HHS) noted they have improved partnering with community-based homecare organizations:

Box I Examples of patient, family, and provider feedback on participation in the collaborative

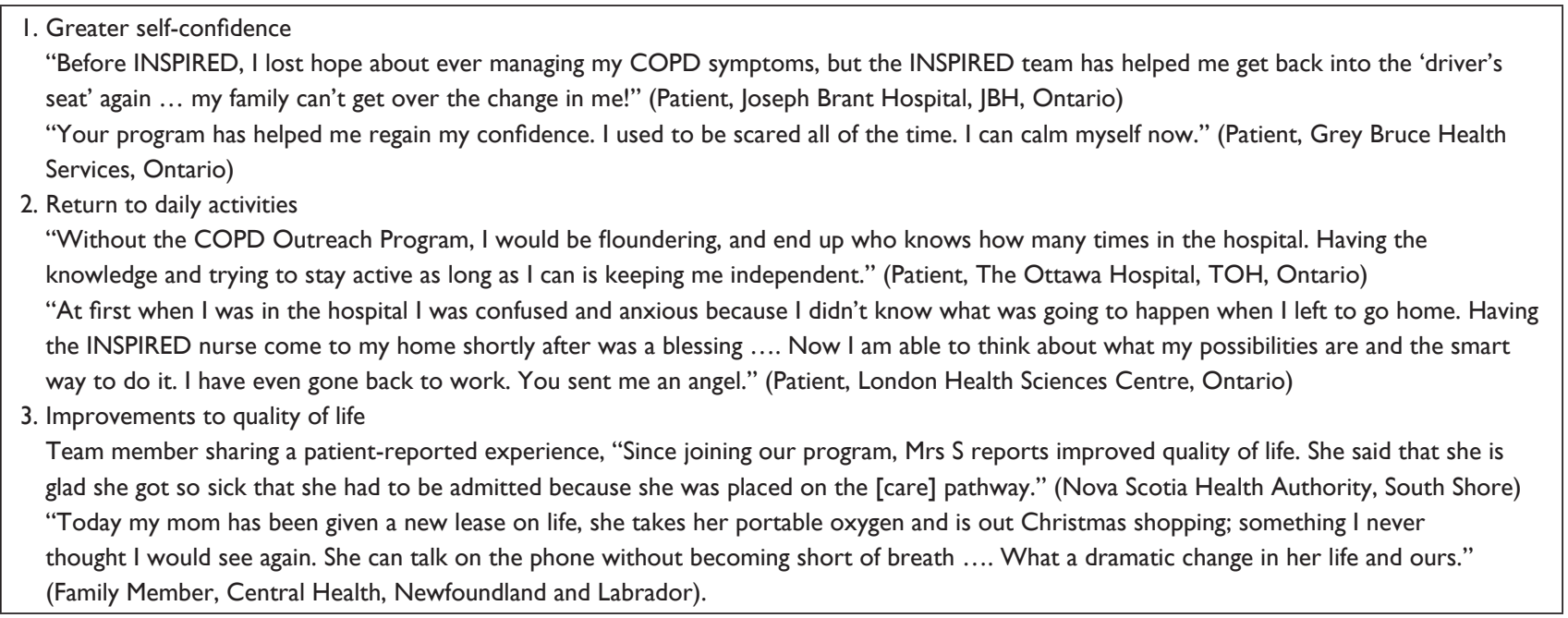


The collaborative provided an opportunity for development and testing of an innovative partnership model between the hospital and community-based partners to ensure that the right care is delivered in the right place at the right time.

HHS also reported (from the first 7 months of their program) that $>90 \%$ of patients meeting enrollment criteria $(n=24)$ received a consultation from Respirology and a medication action plan and that there was consistent use of MRC scores to provide an understanding of "respiratory disability" that assisted with planning for and provision of services after discharge.

\section{Efficiency of care}

Teams' ability to enroll and evaluate outcomes in a timely fashion was quite variable. Median (interquartile range) number of patients enrolled by the 19 teams was 31 (16-83). We highlight two teams from provinces 2,000 miles apart who achieved early very positive results against the four quality dimensions despite differences in contexts and contrasting long-term sustainability: The Ottawa Hospital, Ontario, is a large urban academic hospital, while Providence Health Care, based in Vancouver, is the largest Catholic health care organization in Canada, delivering tertiary/ quaternary care, teaching, and research to some of society's most vulnerable patient populations.

\section{Case I. The Ottawa Hospital}

In Ontario, over 290,000 people live with some forms of COPD. ${ }^{23}$ In a calendar year (2013-2014), patient hospitalizations related to COPD reached $>27,000$ with average length of stay of 6.4 bed days. ${ }^{24}$ As part of the collaborative, the Ottawa Hospital COPD Outreach program sought to improve care quality, effectiveness, and efficiency for patients living with advanced COPD, as well as their families.

Among outcomes, the Ottawa Hospital achieved the following quality-of-care gains for 87 patients enrolled over 10 months (from November 2014) in their pre-post analysis: $70 \%$ reduction in 30 -day readmission rates, $62 \%$ reduction in ED visits, $45 \%$ reduction in hospital admissions, $18 \%$ reduction in average length of stay, and a cost aversion related to a fall in facility reliance of $\$ 250,000$ (after 10-month program cost deduction of $\$ 75,000)$. Of the target population, $100 \%$ received an action plan and $100 \%$ of smokers received smoking cessation counseling. While $55 \%$ of enrolled patients were not interested in referral to community services, $41 \%$ were referred to pulmonary rehabilitation and $4 \%$ to palliative care.
Sustainability: The Ottawa Hospital team is now funded on a long-term basis to continue their initiative related to INSPIRED.

\section{Case II. Providence Health Care}

In British Columbia, 95,000 people live with COPD. ${ }^{23}$ Patient hospitalizations reached $>9,200$ in 2013-2014, with an average length of stay for COPD of 7.8 bed days. ${ }^{24}$ The Providence COPD Outreach Program initiative focused on building bridges between hospital and community care to help patients with COPD stay as healthy as possible by providing individualized care for COPD patients with complex needs. Through their involvement in the collaborative, they appointed an SCP to their team. The top three initial goals of their adaption of INSPIRED were to reduce 30-day readmissions from $17 \%$ to $10 \%$, increase ACP rates from $20 \%$ to $80 \%$, and improve quality-of-life scores by $20 \%$. They also hoped to provide individual action plans and advice regarding smoking cessation and medication usage.

The Providence team enrolled 121 patients between April 1, 2015, and May 19, 2016. Of these, 25 were still enrolled as of May 19, and eight had died, with unavailable data for two patients leaving 86 patients available for analysis $84 \%$ of whom were classified as Global Initiative for Chronic Obstructive Lung Disease (GOLD) stage 3 or 4 (severe or very severe COPD) and 63\% active smokers.

While they fell short of their ACP rate (target $80 \%$, actual $61 \%$ ), this was a major improvement. Target improvements in quality-of-life scores were seen where these data were available (from $n=32$ patients) adjudged by median changes in the CAT score of $28 \%$ (from 19.5 to 15.3 ), Patient Health Questionnaire 4-item scale (PHQ-4, 33\%) from 5.1 to 3.4, and in a 5-point Likert scale for anxiety (28\% from 4.1 to 3.0$)$. A total of $49 \%$ of patients were referred to and $35 \%$ enrolled in pulmonary rehabilitation; confirmation of COPD through spirometry was achieved for $100 \%$, and $82 \%$ of patients received an action plan.

Providence analyzed ED visits and admissions by timeadjusted monthly comparisons during a year prior to and after enrollment, reporting falls of $30 \%$ for admissions and $4 \%$ for ED visits, respectively, representing a cost aversion of $\$ 262,557$, after consideration of program costs $(\$ 50,000)$.

Sustainability: At the time of writing, and despite these achievements, Providence Health Care has not provided funding to allow the local team to sustain the program. The program is no longer active.

Both teams benefited from engaged leadership and frontline staff from the outset of their initiatives, as well 
as dedicated resources to redesign existing services to better meet the needs of their patient populations. Overall, 17 teams reported fewer ED visits and hospitalizations with early reductions as high as $80 \%$ lowering to $40 \%-50 \%$ in 6- and then 12-month pre-post analyses (as in Halifax). ${ }^{7}$ We hope to report these data in detail in due course. For the various participating institutions, bed days freed up by INSPIRED allows earlier access for others with higher acuity needs, thus effecting a more efficient use of a costly finite resource.

\section{Appropriateness of care}

Most teams reported gaining deeper knowledge around the complexities of COPD care, thereby optimizing patient care through action plans, self-management support, psychosocial support, ACP, and coordinating community partnerships. Almost all teams gained a better perspective of their patients' lived experiences and the challenges inherent in managing a complex chronic condition. As an example, Central Health in Newfoundland and Labrador engaged all 19 of their enrolled patients in ACP and end-of-life goals of care discussions. Teams were also better able to coordinate community resources and provide greater support to patients as they began to understand existing patient care pathways. The NSHA South Shore team ensured that all 16 of their enrolled patients received a Victoria Order of Nurses (VON) visit post-discharge from the hospital and follow-up appointments with their primary care physicians at the Asthma and COPD Education Centre.

\section{Implementation barriers}

We describe in some detail elsewhere ${ }^{17}$ the facilitators to program implementation and focus herein on the challenges. The majority of programs reported staff problems that included time conflicts, where employees were unable to dedicate adequate time to the improvement efforts, lack of adherence to the strategies being implemented, lack of experience/training necessary to facilitate/implement components of planned programs, and lack of commitment to the program. Leadership was the second largest challenge for many. This included stakeholder relationships and cross-organizational partnerships where communication was minimal. The third largest challenge for organizations included patient location (distances involved), patient nonadherence to program strategies, and patient scheduling. Some organizations also indicated difficulty recruiting according to eligibility criteria. Accessing home care and services away from the hospital also proved to be a challenge for others.

\section{Discussion}

Through the leadership of the CFHI, 19 teams were provided with the necessary supports and infrastructure to participate within the year-long collaborative. At the outset of the collaborative, it was not known whether success of INSPIRED seen in Halifax could be emulated on a more widespread basis. Even within the relatively short timeframe of the collaborative, the outcomes and experiences reported by participating teams confirm that a community-based program for the benefit of patients with advanced COPD can be implemented widely and successfully across many jurisdictions. Several teams $(n=15)$ continue to enroll patients to their new INSPIRED-related model of care.

More specifically, this program helped:

1. Facilitate adoption and spread of best practices across Canada

Teams modeled their COPD interventions after the INSPIRED model of care, for which there have been demonstrable outcomes on clinical quality and patient experience over the past 6 years. ${ }^{7,25}$ The burdens and costs of living with COPD are well documented and provided a strong incentive for health care delivery organizations to take part in the collaborative and achieve improvements in this area through adaptions of the INSPIRED model. CFHI provided the necessary platform ${ }^{13}$ to help spread this approach nationally.

2. Support health system leaders in achieving meaningful outcomes in patient-centered, cost-effective, timely, and efficient care

The approach to measurement and evaluation needs to be robust and tightly aligned to the implemented intervention. ${ }^{26}$ As a result, the program facilitated the collection of a small number of process and outcome measures which teams were easily able to collect, report on, and use for clinical decision-making. Given that teams were able to adapt the INSPIRED interventions to local context, they had flexibility to adapt the measurement approach to align with their interventions (eg, by selecting relevant appropriateness of care measures and avoiding tools not relevant to their patient population or context). The program leveraged lessons learned from the original INSPIRED team to innovate on ways to effectively measure change. An emerging body of literature supports the need for follow-up services extending beyond the 30-day period following hospitalization. ${ }^{27}$ Teams tracked outcomes for longer, and early results align with this view.

3. Support teams in developing ongoing commitment to quality improvement to deliver enhanced quality of care 
The collaborative supported the successful implementation of evidence-based practices by building improvement teams' capacity for improvement, thereby enabling them to understand essential quality improvement and implementation science principles on measurement and evaluation, use of plan-do-study-act cycles, and change management. Teams also benefited from ongoing coaching support from a network of expert faculty members and program staff. This technical assistance is increasingly recognized as essential to support teams in creating change within their organizations. ${ }^{28}$ The top two major barriers to implementation mandate investment in resources for employees of organizations, in facilitating programs and strategies (reflecting staff-related issues), and effective communication strategies in project planning and facilitation.

\section{Limitations}

Despite generating early positive results, we acknowledge some limitations tied to the collaborative design and rollout. First, the lessons learned may not be generalizable to all jurisdictions in Canada or elsewhere or to care of patients with more mild forms of COPD. Teams had the flexibility to adapt the original INSPIRED model of care to their local settings making it more difficult to isolate the specific impact of interventions and to ensure fidelity (ie, whether the intervention was delivered as intended ${ }^{19}$ ). Further testing across different contexts could likely help identify the most impactful and transferable features of the INSPIRED model for advanced COPD patients. The broader issue of overall improvements to medical care (beyond the care of patients with advanced COPD) and sustainability of innovation nationally are two important considerations but beyond the scope of this initiative.

We accept that the impact of the collaborative would be bolstered by knowledge of the numbers of patients with advanced COPD in each participating location. We have a nascent understanding of this issue in Canada. Moreover, local or regional efforts might be better focused on determining those patients with COPD who are high users of health care. The Canadian Institute of Health Information (CIHI) has reported recently on this issue for Alberta. ${ }^{29}$ Understanding reasons behind high facility reliance for these patients and factors that drive high costs of care ${ }^{30}$ should inform more effective preventative strategies to the benefit of both patients and the health care system.

Current availability of data and infrastructure across health care organizations does not enable teams to collect and analyze data in a consistent way, thereby making it challenging to aggregate results as part of the CFHI-led summative evaluation. Using common data sets and operational definitions from the outset of the program may mitigate this challenge in future collaboratives. Nevertheless, we believe that our findings can help to augment the evidence base related to spreading evidence-based practices and yield deeper insights as to how to evaluate effectiveness. As expertise in delivery and evaluation of QICs grow, we anticipate improvements to COPD care across Canada and beyond.

At the time of writing, we can report that Nova Scotia Health Authority has recently approved additional funding to allow first steps of scale-up of the INSPIRED approach within the province. Several other provinces are considering more widespread implementation of an INSPIRED-like approach based on the early positive outcomes of this QIC and the substantial potential cost benefits. ${ }^{11}$ In addition, the CFHI will release a call in July 2017 for a second QIC involving province-level scale-up.

\section{Conclusion}

Quality-of-care gains are achievable in the short term among different teams across diverse geographical and social context, but sustainability of gains requires longer term planning and committed support. A well-designed, adequately funded public-private partnership can deliver widespread beneficial outcomes for patients and families living with advanced COPD with major implications for health care delivery and system redesign across Canada.

\section{Acknowledgments}

The INSPIRED approaches to COPD care collaborative was hosted by the Canadian Foundation for Healthcare Improvement (CFHI) with arm's length funding from Boehringer Ingelheim Canada Ltd (BICL). CFHI is a not-for-profit organization funded by the Government of Canada. The views expressed herein do not represent those of the Government of Canada. The authors thank Maureen O'Neil O.C. (President) and Stephen Samis (Vice-President) of the CFHI as well as the faculty for their support for the implementation and delivery of the INSPIRED COPD collaborative and the teams who accepted this call to action to join the collaborative. We thank Meghan Rossiter, Kirby Kirvan, and Catherine Giroux of CFHI for their dedication to the successful delivery of the multiple components of the collaborative and/or its evaluation. We acknowledge Catherine Simpson $\mathrm{PhD}$ for her review of an earlier draft of this manuscript. We gratefully acknowledge the leadership of BICL for 
their arm's length funding and in-kind support for teams enrolled in the INSPIRED COPD collaborative.

\section{Disclosure}

JV is and CA was employed by CFHI; GMR is a consultant working with CFHI. The authors report no other conflicts of interest in this work.

\section{References}

1. Canadian Institute for Health Information (CIHI). National Health Expenditure Trends, 1975 to 2012; 2012. Available from: https://secure. cihi.ca/free_products/NHEXTrendsReport2012EN.pdf. Accessed June 30, 2017.

2. Canadian Institute for Health Information (CIHI) [webpage on the Internet]. All-Cause Readmission to Acute Care and Return to the Emergency Department; 2012. Available from: https://secure.cihi.ca/ estore/productFamily.htm?pf $=\mathrm{PFC} 1823 \&$ lang $=$ en\&media $=0$. Accessed June 30, 2017.

3. Mittmann N, Kuramoto L, Seung SJ, Haddon JM, Bradley-Kennedy C, Fitzgerald JM. The cost of moderate and severe COPD exacerbations to the Canadian healthcare system. Respir Med. 2008;102(3):413-421.

4. Rocker GM, Simpson AC, Horton R. Palliative care in advanced lung disease: the challenge of integrating palliation into everyday care. Chest. 2015;148(3):801-809.

5. Rocker GM, Dodek PM, Heyland DK; Canadian Researchers at the End of Life Network. Toward optimal end-of-life care for patients with advanced chronic obstructive pulmonary disease: insights from a multicentre study. Can Respir J. 2008;15(5):249-254.

6. Rocker GM, Cook D. 'INSPIRED' approaches to better care for patients with advanced COPD. Clin Invest Med. 2013;36(3):E114-E120.

7. Rocker GM, Verma JY. 'INSPIRED' COPD outreach program: doing the right things right. Clin Invest Med. 2014;37(5):E311-E319.

8. Bourbeau J, Julien M, Maltais F, et al; Chronic Obstructive Pulmonary Disease axis of the Respiratory Network Fonds de la Recherche en Santé du Québec. Reduction of hospital utilization in patients with chronic obstructive pulmonary disease: a disease-specific self-management intervention. Arch Intern Med. 2003;163(5):585-591.

9. Effing T, Monninkhof EM, van der Valk PD, et al. Self-management education for patients with chronic obstructive pulmonary disease. Cochrane Database Syst Rev. 2007;(4):CD002990.

10. Gardiner C, Gott M, Small N, et al. Living with advanced chronic obstructive pulmonary disease: patients concerns regarding death and dying. Palliat Med. 2009;23(8):691-697.

11. Rocker GM, Verma JY, Demmons J, Mittmann N. Number needed to... Save? Clin Invest Med. 2015;38(1):E11-E14.

12. Smetanin P, McNeil D, Burger C. Modelling the INSPIRED COPD Outreach Program: National and Provincial Analysis Final Report. RiskAnalytica; 2016. Available from: http://www.cfhi-fcass.ca/ sf-docs/default-source/documents/inspired/inspired-riskanalytica-e. pdf?sfvrsn=2. Accessed June 30, 2017.

13. Ministry of Health (Canada). Report of the Advisory Panel on Healthcare Innovation; 2015. Available from: https://opto.ca/sites/ default/files/resources/documents/report-healthcare-innovation-rapportsoins-eng.pdf. Accessed November 11, 2016.
14. Canadian Foundation for Healthcare Improvement (CFHI) [webpage on the Internet]. INSPIRED COPD Collaborative; 2016. Available from: http://www.cfhi-fcass.ca/WhatWeDo/inspired-approaches-to-copd. Accessed June 30, 2017.

15. Canadian Foundation for Healthcare Improvement [webpage on the Internet]. CFHI Improvement ModelTM; 2014. Ottawa, ON: Canadian Foundation for Healthcare Improvement. Available from: http://www. cfhi-fcass.ca/WhatWeDo/Collaborations/OurApproach.aspx. Accessed June 30, 2017.

16. Gillis D, Demmons J, Rocker G. Expanding The INSPIRED COPD outreach program to the emergency department: a feasibility assessment. Int J Chron Obstruct Pulmon Dis. 2017;12:1597-1604.

17. Verma JY, Amar C, Sibbald S, Rocker GM. Improving care for advanced COPD through practice change: experiences of participation in a Canadian spread collaborative. Chron Respir Dis. Epub 2017 Jan 1.

18. Parry C, Mahoney E, Chalmers SA, Coleman EA. Assessing the quality of transitional care: further applications of the care transitions measure. Med Care. 2008;46(3):317-322.

19. Moore GF, Audrey S, Barker M, et al. Process evaluation of complex interventions: Medical Research Council guidance. BMJ. 2015; 350:h1258.

20. Rocker GM, Simpson AC, Young J, et al. Opioid therapy for refractory dyspnea in patients with advanced chronic obstructive pulmonary disease: patients' experiences and outcomes. CMAJ Open. 2013;1(1): E27-E36.

21. Hyland ME, Jones RC, Hanney KE. The Lung Information Needs Questionnaire: development, preliminary validation and findings. Respir Med. 2006;100(10):1807-1816.

22. Jones PW, Harding G, Berry P, Wiklund I, Chen WH, Kline Leidy N. Development and first validation of the COPD Assessment Test. Eur Respir J. 2009;34(3):648-654.

23. Statistics Canada [webpage on the Internet]. Chronic Obstructive Pulmonary Disease by Sex, by Province and Territory; 2016. Available from: http://www.statcan.gc.ca/tables-tableaux/sum-som/101/cst01/ health105a-eng.htm. Accessed June 30, 2017.

24. CIHI [webpage on the Internet]. Inpatient Hospitalizations, Surgeries and Childbirth Indicators; 2015. Available from: https://secure.cihi.ca/ estore/productFamily.htm?locale=en\&pf=PFC2805. Accessed June 30, 2017.

25. Rocker G, Downar J, Morrison RS. Palliative care for chronic illness: driving change. CMAJ. 2016;188(17-18):E493-E498.

26. Ovretveit J, Bate P, Cleary P, et al. Quality collaboratives: lessons from research. Qual Saf Health Care. 2002;11(4):345-351.

27. Jonkman NH, Westland $\mathrm{H}$, Trappenburg JC, et al. Characteristics of effective self-management interventions in patients with COPD: individual patient data meta-analysis. Eur Respir J. 2016;48(1):55-68.

28. Nadeem E, Olin SS, Hill LC, Hoagwood KE, Horwitz SM. Understanding the components of quality improvement collaboratives: a systematic literature review. Milbank Q. 2013;9(2):354-394.

29. Canadian Institute of Health Information. COPD in Alberta: Examining the Characteristics and Health Care Use of High Users. Ottawa, ON: Canadian Institute of Health Information; 2017.

30. Mulpuru S, McKay J, Ronksley PE, Thavorn K, Kobewka DM, Forster AJ. Factors contributing to high-cost hospital care for patients with COPD. Int J Chron Obstruct Pulmon Dis. 2017;12:989-995.
International Journal of COPD

\section{Publish your work in this journal}

The International Journal of COPD is an international, peer-reviewed journal of therapeutics and pharmacology focusing on concise rapid reporting of clinical studies and reviews in COPD. Special focus is given to the pathophysiological processes underlying the disease, intervention programs, patient focused education, and self management protocols.

\section{Dovepress}

This journal is indexed on PubMed Central, MedLine and CAS. The manuscript management system is completely online and includes a very quick and fair peer-review system, which is all easy to use. Visit $\mathrm{http}: / / \mathrm{www}$.dovepress.com/testimonials.php to read real quotes from published authors. 